\title{
Langages formels dans la machine abstraite biochimique BIOCHAM
}

\author{
Laurence Calzone - Nathalie Chabrier-Rivier - François Fages - \\ Loïc Fosse - Sylvain Soliman
}

INRIA Rocquencourt - Projet CONTRAINTES

Domaine de Voluceau, Rocquencourt, BP 105

78153 LE CHESNAY CEDEX

\{Prenom.Nom\}@inria.fr

RÉSUMÉ. Le développement de langages formels pour modéliser les systèmes biologiques ouvre la voie à la conception de nouveaux outils de raisonnement automatique destinés au biologiste modélisateur. La machine abstraite biochimique BIOCHAM est un environnement logiciel qui offre un langage simple de règles pour modéliser des interactions biomoléculaires, et un langage puissant fondé sur la logique temporelle pour formaliser les propriétés biologiques du système. En s'appuyant sur ces deux langages formels, il devient possible d'utiliser des techniques d'apprentissage automatique pour inférer de nouvelles règles de réaction, estimer les valeurs des paramètres cinétiques, et corriger ou compléter les modèles semi-automatiquement. Dans cet article, nous décrivons les langages implantés dans BIOCHAM et illustrons l'utilisation du système d'apprentissage automatique sur un modèle simple du contrôle du cycle cellulaire.

ABSTRACT. With the advent of formal languages for modeling biological systems, the design of automated reasoning tools to assist the biologist becomes possible. The biochemical abstract machine BIOCHAM software environment offers a rule-based language to model bio-molecular interactions and a powerful temporal logic based language to formalize the biological properties of the system. Building on these two formal languages, machine learning techniques can be used to infer new molecular interaction rules from temporal properties, or to estimate kinetic parameter values, in order to semi-automatically correct or complete models from observed biological properties of the system. In this article we describe the formal languages of BIOCHAM and illustrate, on a simple cell cycle control model, the use of the machine learning system.

MOTS-CLÉS : Biologie des systèmes, logique temporelle, apprentissage, cycle cellulaire

KEYWORDS: Systems biology, temporal logics, machine learning, cell cycle

$1^{\text {re }}$ soumission à Technique et science informatiques, le 7 octobre 2005 


\section{Introduction}

La production en masse de données post-génomiques, telles que l'expression des ARN, la production de protéines et les interactions protéine-protéine, donne plus d'acuité au besoin, et aux difficultés, qu'il y a à raisonner à l'échelle d'un système complexe d'interactions biomoléculaires. Les connaissances concernant les réseaux d'influence géniques et les réseaux d'interaction protéiques sont intégrées dans des bases de données comme KEGG (Kanehisa et al., 2000), BioCyc (Keseler et al., 2005), ... et présentées généralement sous forme de diagrammes annotés. Les outils comme BioSpice (Giaever et al., 2002), Copasi (VBI n.d.), GON, E-cell (Tomita et $a l ., 2000)$ etc. ont été développés pour faire des simulations numériques lorsque les données cinétiques sont connues.

Cependant, au delà de la simulation, la possibilité de faire des calculs symboliques sur les réseaux d'interactions moléculaires ouvre la voie à la réalisation de nouveaux outils de raisonnement automatique destinés au biologiste modélisateur. Notre projet avec la Machine Abstraite Biochimique BIOCHAM ${ }^{1}$ (Fages et al., 2004), qui a débuté en 2002, est une tentative dans cette direction. BIOCHAM est fondé sur un langage de composés moléculaires et de règles de réaction, qui fournit des sémantiques précises aux diagrammes d'interactions biomoléculaires, à trois niveaux d'abstraction :

1) la sémantique Booléenne, qui permet de raisonner sur la présence ou l'absence des molécules, associe à un ensemble de règles de réaction un système de transitions concurrent asynchrone ;

2) la sémantique des concentrations, qui permet de raisonner sur la dynamique continue du système, associe des valeurs réelles aux molécules représentant leur concentration, et associe aux règles un système d'équations différentielles (non linéaires) du premier ordre ;

3) la sémantique des populations, qui permet de raisonner sur les petits nombres de molécules, associe aux molécules une valeur entière représentant leur quantité, et associe aux règles une chaîne de Markov en temps continu.

Dans les trois cas, nous utilisons la logique temporelle comme langage de formalisation des propriétés biologiques du système. Dans la sémantique Booléenne, la logique des arbres de calcul CTL (Clarke et al., 1999) permet d'exprimer un large spectre de propriétés biologiques telles que l'accessibilité, les points de passage obligé, la stabilité ou les oscillations (Chabrier et al., 2003). Nous avons montré le passage à l'échelle des algorithmes de model-checking dans ce contexte, sur un modèle Booléen du contrôle du cycle cellulaire des mammifères, développé d'après le diagramme de Kohn (Kohn, 1999) et impliquant environ 500 variables et 800 règles de réaction (Chabrier-Rivier et al., 2004a). Dans la sémantique des concentrations, la logique LTL (Clarke et al., 1999) avec contraintes numériques permet d'exprimer des propriétés temporelles quantitatives. Dans la sémantique des populations, la logique

1. BIOCHAM est un logiciel distribué sous licence GPL à l'adresse http ://contraintes.inria.fr/BIOCHAM/ 
PCTL (Hansson et al., 1994, Kwiatkowska et al., 2004) avec contraintes numériques permet d'exprimer les probabilités de réalisation de propriétés temporelles quantitatives.

La vérification automatique des propriétés d'un système biologique formalisées en logique temporelle, permet non seulement d'interroger un modèle, mais aussi d'aider à le corriger, en prenant ces propriétés comme une spécification et en cherchant automatiquement des règles et des paramètres permettant de la satisfaire (Calzone et al., 2005). Dans cet article, nous décrivons les deux méthodes d'apprentissage implantées dans BIOCHAM. La première, qui utilise la sémantique Booléenne, concerne la recherche de règles de réaction à ajouter ou retirer au modèle de façon à satisfaire une spécification CTL. La seconde, qui utilise la sémantique des concentrations, concerne la recherche de paramètres cinétiques permettant de satisfaire une spécification LTL avec contraintes. Les deux méthodes sont illustrées sur un modèle simplifié du contrôle du cycle cellulaire.

\subsection{Travaux reliés}

Les travaux pionniers de Regev, Silverman and Shapiro (2001), sur l'utilisation du $\pi$-calcul de Milner pour modéliser les voies de signalisation dans la cellule, ont été la source d'inspiration de nombreux travaux ultérieurs dans la lignée des calculs de processus (Cardelli, 2004, Regev et al., 2004, Danos et al., 2004) et de leurs extensions stochastiques (Phillips et al., to appear). Le langage de règles de BIOCHAM se veut une simplification de cette démarche utilisant les calculs de processus, au profit d'un formalisme simple de règles de réaction, qui d'une part est très naturel pour le biologiste, et d'autre part se prête mieux aux techniques d'analyse par model-checking. Dans le cas Booléen, ce langage est similaire aux règles de réécriture de Pathway Logic implantées dans le système Maude (Eker et al., 2002). L'utilisation de la logique temporelle et des techniques de model-checking en biologie des systèmes est une idée récente, elle apparait dans (Eker et al., 2002, Chabrier et al., 2003) pour les modèles Booléens, dans (Bernot et al., 2004, Batt et al., 2004, Calder et al., 2005) pour les modèles discrets et dans (Antoniotti et al., 2003) pour les modèles continus.

Les techniques d'apprentissage automatique comme la programmation logique inductive (Muggleton, 1995) ou la programmation génétique, ont été utilisées pour inférer des fonctions de gènes (Bryant et al., 2001), des descriptions de voies métaboliques (Angelopoulos et al., 2002a, Angelopoulos et al., 2002b, Koza et al., 2001) ou des interactions entre gènes (Bernot et al., 2004). Nos travaux se rapprochent du domaine de la découverte scientifique qualitative et numérique (Langley et al., 1987) et de la révision de théories (Todorovski et al., 2001, de Raedt, 1992). Cependant, l'apprentissage à partir de formules temporelles est un sujet nouveau, que ce soit du point de vue de l'apprentissage automatique, ou de la biologie des systèmes. 


\section{Langage de description de processus biomoléculaires}

Les règles de réaction BIOCHAM représentent des réactions entre des objets formels représentant des composés chimiques ou biochimiques, qui s'étendent des petites molécules aux gènes et aux protéines.

La syntaxe des molécules et des réactions peut être donnée par la grammaire (simplifiée) suivante :

$\begin{array}{ll}\text { objet } & =\text { molecule } \mid \text { molecule }: \text { lieu } \\ \text { molecule } & =\text { nom } \mid \text { molecule-molecule } \mid \text { molecule } \sim\{\text { nom,...,nom }\} \\ \text { reaction } & =\text { solution }=>\text { solution } \mid \text { cinetique for } \text { solution }=>\text { solution } \\ \text { solution } & =-\mid \text { objet } \mid \text { nombre*objet } \mid \text { solution }+ \text { solution }\end{array}$

L'objet de base est un composé moléculaire. Il peut être localisé avec l'opérateur “:” en indiquant un lieu, qui est un simple nom représentant un compartiment tel que le noyau, le cytoplasme, la membrane, etc... L'opérateur de liaison "_" sert à représenter la complexation ou d'autres formes de liaison de molécules. L'opérateur de modification " " permet d'attacher un ensemble de modifications à une molécule, tel que l'ensemble des sites phosphorylés d'une protéine. Pour les règles de réaction, les abréviations suivantes peuvent être utilisées : $A=[C]=>B$ pour la règle $A+C=>$ $B+C$ avec le catalyseur $C$, et $A<=>B$ pour les deux réactions symétriques. Par exemple, $M+N \Rightarrow M-N$ représente une règle de complexation, $M=$ $[N]=>M \sim\{s\}$ une règle de phosphorylation catalysée par une kinase $N$, et $M: L=>M: L^{\prime}$ représente une règle de transport de $M$, de $L$ en $L^{\prime}$. Dans tous les cas, les règles peuvent être données avec une expression cinétique, correspondant par exemple à la loi d'action de masse.

BIOCHAM possède aussi un langage de schémas avec contraintes que nous ne décrivons pas ici, et qui est utilisé pour spécifier de façon concise des ensembles de molécules et de règles de réaction, ou pour indiquer la forme des règles à apprendre.

Exemple 1 Le cycle cellulaire décrit le mécanisme bio-moléculaire par lequel une cellule est amenée à se diviser et proliférer. Il s'agit d'une séquence d'événements qui inclue la réplication de l'ADN (phase S), la mitose (phase M) et l'apparition et la disparition successives de diverses protéines comme les cyclines. Pour être actives, ces cyclines s'associent à d'autres protéines, des kinases cycline-dépendantes. Les complexes ainsi formés contrôlent le déroulement du cycle.

L'exemple BIOCHAM ci-dessous est un modèle simple du cycle cellulaire tiré de (Tyson, 1991). Il rend compte des interactions existant entre la kinase Cdc2 et une cycline. Les deux molécules se complexent en un hétérodimère qui s'active et s'inactive par divers mécanismes de phosphorylation et de déphosphorylation. La forme active du complexe, Cdc2-Cyclin $\{\mathrm{p} 1\}$ (aussi appelé MPF pour M-phase Promoting Factor), joue un rôle central dans le cycle mitotique : lorsqu'elle est présente la cellule est en phase $M$. 


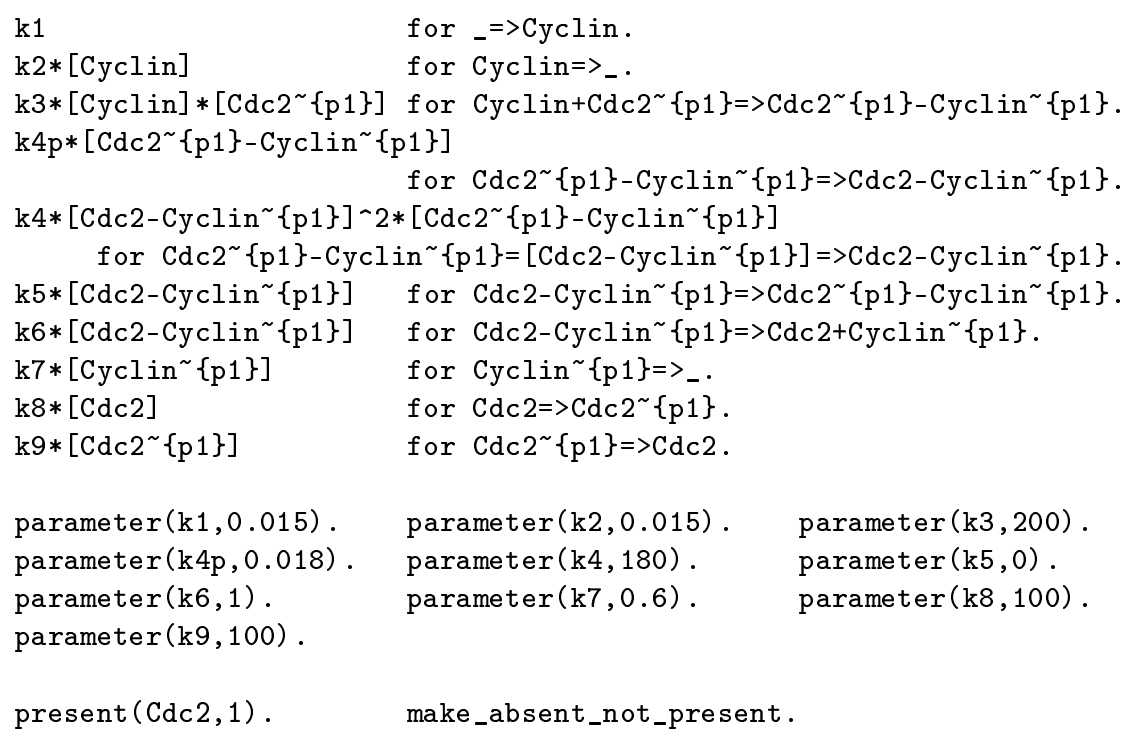

Les dernières instructions définissent l'état initial du modèle, dans lequel Cdc2 est présent (avec une concentration initiale égale à 1) et les autres composés sont absents (concentration 0).

La sémantique des règles BIOCHAM est définie à trois niveaux d'abstraction qui correspondent à trois formes de raisonnement sur les systèmes biologiques et à trois sémantiques formelles du langage des règles : la sémantique Booléenne, la sémantique des concentrations en nombres réels, et la sémantique des populations de molécules en nombres entiers.

\subsection{Sémantique Booléenne}

Dans la sémantique Booléenne, on associe à chaque objet une variable Booléenne qui représente sa présence ou absence dans le système, et les règles de réaction sont interprétées comme un système de transitions concurrent asynchrone. Une règle comme $A+B=>C+D$ définit quatre transitions possibles qui correspondent à la consommation totale ou non des réactifs $A$ et $B$ par la réaction. Pour que la règle soit applicable, les molécules $A$ et $B$ doivent être présentes dans l'état courant du système. Dans l'état suivant les molécules $C$ et $D$ sont présentes tandis que les molécules $A$ et $B$ peuvent être absentes (consommation totale) ou présentes (consommation partielle). Ceci se fait de façon non-déterministe afin de rendre compte de tous les comportements possibles. Le système de transitions est asynchrone dans le sens où une seule transition est appliquée à la fois, ce qui permet de représenter les phénomènes biologiques fondamentaux de masquage d'une réaction par une autre, comme par exemple dans le cas de certaines inhibitions. A l'inverse, l'hypothèse de synchronie (dans la- 


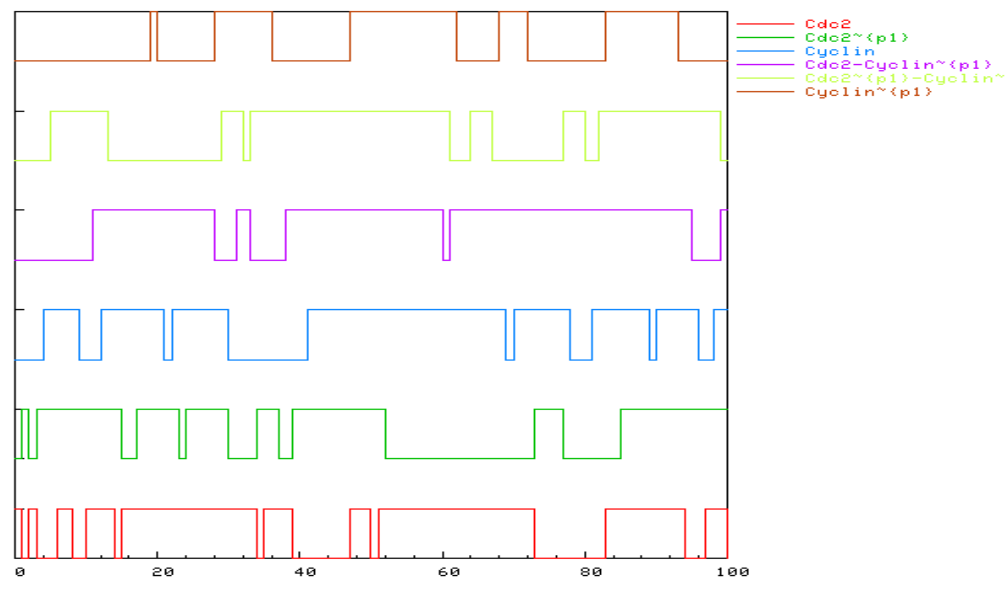

Figure 1. Exemple de trace d'une simulation Booléenne du modèle du cycle cellulaire de l'exemple 1.

quelle toutes les règles applicables se déclenchent en même temps) ne permettrait pas de représenter correctement les phénomènes de compétition entre réactions.

Formellement, la sémantique Booléenne d'un modèle BIOCHAM est définie par une structure de Kripke $K=(S, R)$, où $S$ est l'ensemble des états définis par le vecteur de variables Booléennes, $R \subseteq S \times S$ est la relation de transition entre états supposée totale (c'est-à-dire que pour tout état $s \in S$, il existe un état $s^{\prime} \in S$ tel que $\left.\left(s, s^{\prime}\right) \in R\right)$. Lorsque la relation de transition provenant uniquement des réactions BIOCHAM n'est pas totale, elle est automatiquement complétée par une transition qui ajoute une boucle sur les états n'ayant pas de successeur. Un chemin dans $K$ partant de l'état $s_{0}$ est une suite infinie d'états $\pi=s_{0}, s_{1}, \ldots$ telle que $\left(s_{i}, s_{i+1}\right) \in R$ pour tout $i \geq 0 . \pi^{k}$ désignera le chemin $s_{k}, s_{k+1}, \cdots$. L'évolution temporelle du système est modélisée par la succession des étapes de transition, et les différents comportements du système sont modélisés par le choix non-déterministe des transitions. La figure 1 montre une trace de simulation Booléenne du modèle du cycle cellulaire.

\subsection{Sémantique des concentrations}

Dans la sémantique des concentrations, on associe à chaque objet une variable réelle qui représente sa concentration, et les règles sont interprétées par un système d'équations différentielles. Formellement, à un ensemble de règles BIOCHAM $E=$ 


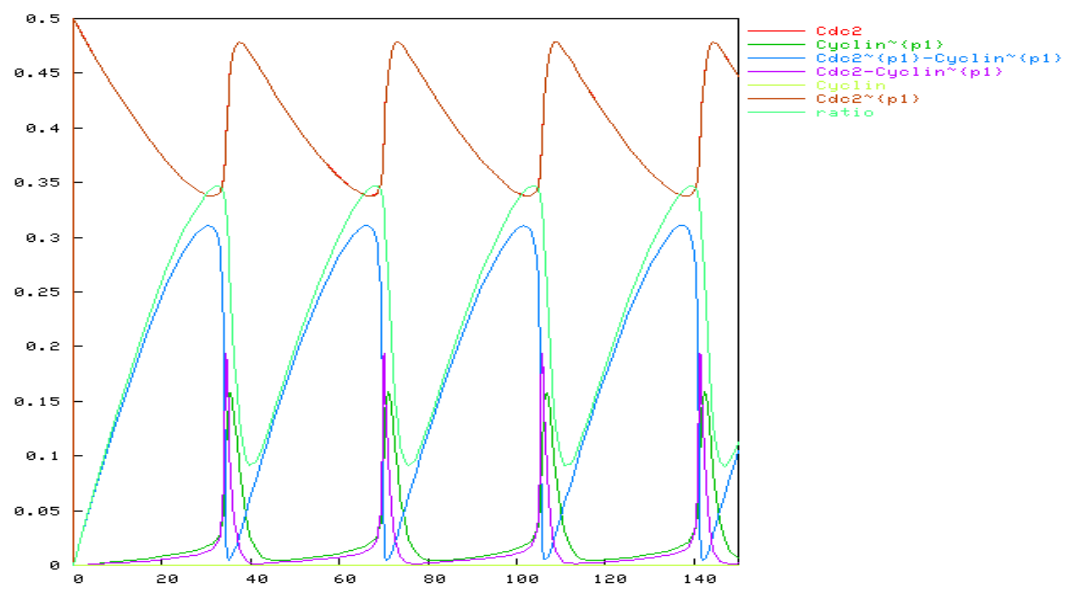

Figure 2. Simulation numérique du modèle du cycle cellulaire de l'exemple 1

$\left\{e_{i} \text { for } S_{i} \Rightarrow S_{i}^{\prime}\right\}_{i=1, \ldots, n}$ sur un ensemble de variables $\left\{x_{1}, \ldots, x_{m}\right\}$, on associe le système d'équations différentielles

$$
d x_{k} / d t=\sum_{i=1}^{n} r_{i}\left(x_{k}\right) * e_{i}-\sum_{j=1}^{n} l_{j}\left(x_{k}\right) * e_{j}
$$

où $r_{i}(x)$ (resp. $l_{i}$ ) est le coefficient stoechiométrique de $x$ dans le membre droit (resp. gauche) de la règle $i$.

Étant donné un état initial défini par les concentrations initiales des objets, les méthodes d'intégration numérique permettent de simuler le comportement du système. La figure 2 illustre par exemple les oscillations des concentrations des protéines décrites dans l'exemple 1.

\subsection{Sémantique des populations}

Dans la sémantique des populations, on associe à chaque objet une variable entière qui représente le nombre de molécules, et les règles sont interprétées par une chaîne de Markov en temps continu dans laquelle les taux de transition sont définis par les expressions cinétiques des règles BIOCHAM.

Les méthodes de simulation stochastique (Gillespie, 1976) calculent des réalisations du processus. Ces courbes sont en général des versions bruitées des courbes obtenues dans la sémantique des concentrations. Cependant dans les modèles faisant intervenir, par exemple, des petits nombres de molécules, des comportements qualitativement différents peuvent apparaître dans les simulations stochastiques. Un exemple 
classique de ce phénomène est le modèle du phage lambda dans lequel un petit nombre de molécules, facteurs de promotion de deux gènes, engendrent un phénomène de multiplication explosive (lyse) après une période d'attente passive plus ou moins longue (lysogénie) (Gibson et al., 2000).

Dans la sémantique stochastique, pour un volume $V$ donné, les concentrations sont donc converties en nombre de molécules ( $N=C \times V \times K, K$ constante d'Avogadro). Les coefficients cinétiques $k$ sont convertis en taux de transition $\tau$ par les formules suivantes :

$-\tau=k$ pour les réactions du type $\mathrm{A}=>\ldots$,

$-\tau=\frac{k}{V \times K}$ pour les réactions du type $\mathrm{A}+\mathrm{B}=>\ldots$,

$-\tau=2 \times \frac{k}{V \times K}$ pour les réactions du type $\mathrm{A}+\mathrm{A}=>\ldots$,

$-\tau=\frac{k}{(V \times K)^{2}}$ pour les réactions du type $\mathrm{A}+\mathrm{B}+\mathrm{C}=>\ldots$,

- etc.

\section{Langage de description de propriétés biologiques}

Le trait le plus original de BIOCHAM est l'utilisation de la logique temporelle comme langage de formalisation des propriétés biologiques d'un modèle. Les logiques CTL Booléenne, LTL et PCTL avec contraintes numériques sont utilisées respectivement dans chacun des trois niveaux sémantiques. Elles permettent de formaliser les propriétés importantes d'un modèle, qui aujourd'hui sont expliquées de façon informelle dans les articles de modélisation. Leur formalisation permet cependant de les considérer comme une spécification devant être préservée lors des opérations de raffinement, de simplification ou de composition de modèles. La possibilité de vérifier automatiquement une telle spécification permet d'envisager la comparaison et la réutilisation de différents modèles de façon beaucoup plus systématique que ce qui se pratique aujourd'hui dans ce domaine.

\subsection{Logique CTL pour la sémantique Booléenne}

La logique temporelle des arbres de calcul (Computation Tree Logic CTL*) (Clarke et al., 1999) est une extension de la logique classique qui permet de raisonner sur un arbre de transitions d'états à l'aide d'opérateurs sur les choix de branches (nondéterminisme) et sur le temps (transitions d'états). Deux quantificateurs de chemins sont introduits pour raisonner sur le non-déterminisme : $A \phi$ qui signifie que $\phi$ est vraie sur tous les chemins, et $E \phi$, pour $\phi$ est vraie sur au moins un chemin. Plusieurs opérateurs temporels sont introduits : $X \phi$ signifiant que $\phi$ est vrai à la transition suivante, $G \phi$ pour $\phi$ est toujours vrai, $F \phi$ pour $\phi$ finit par devenir vrai, $\phi U \psi$ pour $\psi$ finit par devenir vrai et $\phi$ est vrai jusqu'à ce que $\psi$ soit vrai, $\phi W \psi$ pour $\phi$ est vrai jusqu'à ce qu'éventuellement $\psi$ devienne vrai. Dans cette logique, $F \phi$ est équivalent à true $U \phi$, 
$G \phi$ est équivalent à $\phi W$ false et nous avons les propriétés de dualité suivantes : $\neg E F(\phi)=A G(\neg \phi), \neg E \phi U \psi=A \neg \psi W \neg \phi$ et $\neg E \phi W \psi=A \neg \psi U \neg \phi$.

\begin{tabular}{|lll|}
\hline$s \models \alpha$ & ssi & $\alpha$ est une formule propositionnelle et $s \models \alpha$, \\
$s \models E \psi$ & ssi & il existe un chemin $\pi$ partant de $s$ tel que $\pi \models \psi$, \\
$s \models A \psi$ & ssi & pour tout chemin $\pi$ partant de $s, \pi \models \psi$, \\
$\pi \models \phi$ & ssi & $s \models \phi$ où $s$ est l'état de début de $\pi$, \\
$\pi \models X \psi$ & ssi & $\pi^{1} \models \psi$, \\
$\pi \models F \psi$ & ssi & il existe $k \geq 0$ tel que $\pi^{k} \models \psi$, \\
$\pi \models G \psi$ & ssi & pour tout $k \geq 0, \pi^{k} \models \psi$, \\
$\pi \models \psi U \psi^{\prime}$ & ssi & il existe $k \geq 0$ tel que $\pi^{k} \models \psi^{\prime}$ et $\pi^{j} \models \psi$ pour tout $0 \leq j<k$. \\
\hline
\end{tabular}

Tableau 1. Définition inductive de la relation de vérité d'une formule CTL dans un état ou un chemin, dans une structure de Kripke K.

Dans le fragment CTL qui nous intéresse, chaque opérateur temporel doit obligatoirement être précédé d'un quantificateur de chemin et un quantificateur de chemin doit obligatoirement être suivi d'un opérateur temporel. Le tableau 1 définit la relation de vérité d'une formule dans une structure de Kripke $K=(S, R)$ dont les états sont définis par des variables Booléennes. Un modèle BIOCHAM $\mathcal{M}$ est constitué d'un ensemble de règles et d'une spécification (partielle) de l'ensemble des états initiaux. Les règles de $\mathcal{M}$ définissent la structure de Kripke associé au modèle, et on note $\mathcal{M} \models \phi$ si la formule $\phi$ est vrai dans tous les états initiaux spécifiés par $\mathcal{M}$.

\subsubsection{Expressivité}

Comme montré dans (Chabrier et al., 2003), le fragment CTL a une expressivité suffisante pour exprimer un très large éventail de propriétés biologiques :

Sur l'accessibilité. Existe-t-il un chemin pour produire (c-à-d synthétiser, activer etc.) une protéine $P$ ? Cette requête est formalisée par la formule CTL $E F(P)$, abrégée en reachable (P) dans BIOCHAM.

Sur les voies. Est-ce que l'état $Q$ est un point de passage obligé pour atteindre les états où $P \sim\{s\}$ est présent ? La formule correspondante $\neg(E((\neg(Q) U P \sim$ $\{s\})))$ est abrégée en checkpoint $\left(\mathrm{Q}, \mathrm{P}^{\sim}\{\mathbf{s}\}\right)$.

Sur la stationnarité et la stabilité. Est-ce qu'un état (ou un ensemble d'états) de la cellule décrit par une formule $s$ est un état stationnaire (resp. stable) ? $s \Rightarrow$ $E G(s)$ (resp. $s \Rightarrow A G(s)$ ), abrégé en steady (s) (resp. stable(s)).

Sur les oscillations. Le système peut-il exhiber un comportement cyclique sur la présence de $P$ ? $E G((P \Rightarrow E F \neg P) \wedge(\neg P \Rightarrow E F P))$, abrégé en oscil(P). Sans hypothèse d'équité forte, cette formule n'est cependant qu'une approximation car elle est impliquée par l'existence d'oscillations mais n'implique pas l'alternance infinie d'états de types $P$ et $\neg P$. 
Exemple 2 Le modèle du cycle cellulaire précédent vérifie, par exemple, les propriétés suivantes qui expriment que toutes les molécules sont accessibles, que leur activité oscille et qu'il est nécessaire de produire la forme doublement phosphorylée $\mathrm{Cdc2} \sim$ p1\}-Cyclin $\{\mathrm{p} 1\}$ avant l'activation de Cdc2-Cyclin $\{\mathrm{p} 1\}$ :

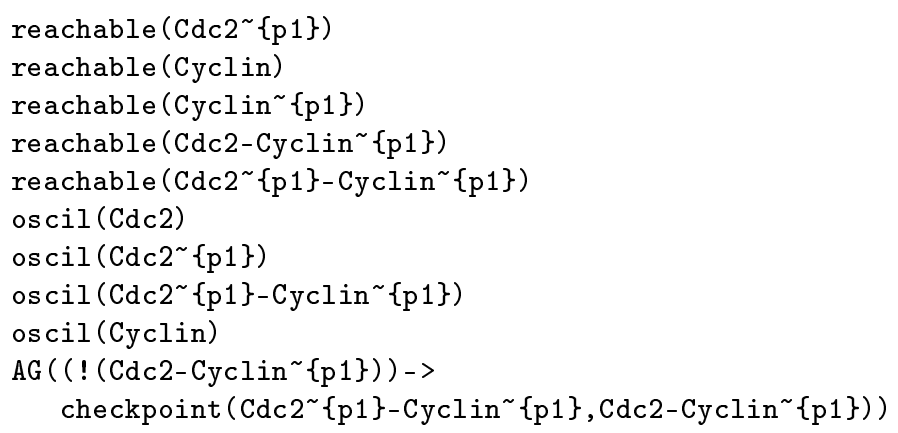

\subsubsection{Algorithme de model-checking symbolique}

Le langage de requête CTL pour les modèles Booléens est implanté en BIOCHAM avec une interface au vérificateur de modèles symbolique NuSMV (Cimatti et al., 2002). Les performances obtenues sur un gros modèle du contrôle du cycle cellulaire suivant la carte de Kohn (Kohn, 1999) comprenant 800 règles et 500 variables sont de l'ordre de quelques dizaines de secondes pour compiler le modèle ou vérifier des formules CTL simples (Chabrier-Rivier et al., 2004a). Cependant ces performances sont en deçà de celles obtenues classiquement par NuSMV avec des modèles fortement structurés pour la vérification de circuits ou de programmes. Les modèles Booléens de BIOCHAM ont la caractéristique d'être très fortement non-déterministes, ce qui peut conduire à des problèmes de performance sur des problèmes de plus petite taille comprenant un grand nombre de voies parallèles, comme par exemple dans le modèle de la voie de signalisation MAPK de (Schoeberl et al., 2002).

\subsection{3. Équivalence CTL de modèles}

Il existe, en général, de nombreuses manières de modéliser un même système, suivant le niveau de détail souhaité et l'information disponible. Même pour une simple réaction enzymatique, il existe déjà deux façons classiques de la coder en BIOCHAM :

- soit en détaillant la formation d'un complexe intermédiaire

$$
\begin{aligned}
& A+B \Leftrightarrow D \\
& D=A+C \\
& \quad-\text { soit directement } \\
& A+B=A+C \text { (que l'on peut aussi noter } B=[A]=>C \text { ) }
\end{aligned}
$$

Le premier choix peut sembler plus simple et naturel pour une conversion en BIOCHAM, alors que le second correspond à la traduction directe de certains modèles numériques à base d'équations différentielles. Les deux propriétés ci-dessous montrent l'équivalence des deux modèles vis-à-vis de certaines propriétés CTL; 
Nous supposerons que le complexe D n'apparaît que dans les deux règles du premier cas, sans quoi la simplification n'est bien sûr pas correcte. Les structures de Kripke associées à ces deux choix définissent deux relations de vérité : $\models_{1}$ et $\models_{2}$.

Proposition 1 (Accessibilité) (Chabrier-Rivier et al., 2004b)

Soit $\phi$ une formule CTL atomique, si $I \models_{2} E F(\phi)$ alors $I \models_{1} E F(\phi)$. De plus, si A et $\mathrm{B}$ n'apparaissent pas négativement (i.e. sous un nombre impair de négations) dans $\phi$ et si D n'apparaît pas du tout dans $\phi$, alors $I \models_{1} E F(\phi)$ implique $I \models_{2} E F(\phi)$.

Proposition 2 (Point de passage obligé) (Chabrier-Rivier et al., 2004b)

Soit $\neg E(\neg \phi U \psi)$ une formule de checkpoint, i.e. $\phi$ et $\psi$ sont des formules atomiques décrivant des états,

si $\mathrm{A}$ et $\mathrm{B}$ n'apparaissent pas négativement dans $\psi$ et $\mathrm{D}$ n'apparaît pas du tout dans $\psi$, alors

$I \models_{2} \neg E(\neg \phi U \psi)$ implique $I \models_{1} \neg E(\neg \phi U \psi)$.

si A et $\mathrm{B}$ n'apparaissent pas négativement dans $\phi$ et $\mathrm{D}$ n'apparaît pas du tout dans $\phi$, alors

$$
I \models_{1} \neg E(\neg \phi U \psi) \text { implique } I \models_{2} \neg E(\neg \phi U \psi) .
$$

\subsection{LTL avec contraintes numériques pour la sémantique des concentrations}

Une extension de la logique temporelle du temps linéaire (Linear Time Logic, LTL) avec contraintes arithmétiques est utilisée pour la sémantique (déterministe) des concentrations moléculaires. Cette approche est similaire à celle décrite dans (Antoniotti et al., 2003) et utilisée dans le projet DARPA BioSpice.

LTL est un fragment de la logique temporelle, qui utilise uniquement les opérateurs temporels (pas les quantificateurs de chemin), et est donc appropriée pour raisonner sur les systèmes déterministes que sont les modèles cinétiques. Dans LTL avec contraintes arithmétiques, les formules atomiques sont des formules de logique du premier ordre avec égalité, inégalité et opérations arithmétiques. Ces formules portent sur les valeurs réelles des concentrations, par exemple $[\mathrm{P}]>\left[\mathrm{P}^{\sim}\{\mathrm{s}\}\right]$, ainsi que celles de leurs dérivées, par exemple $\mathrm{d}([\mathrm{P}]) / \mathrm{dt}<0$.

\subsubsection{Expressivité}

Les propriétés d'accessibilité sont formalisées par l'opérateur $F$. Par exemple, la propriété que la concentration de la protéine $P$ phosphorylée sur $s$ peut être dépassée par celle de la protéine $P$, s'exprime par la formule $F([P]>[P \sim\{s\}])$. La propriété que la concentration de $P$ atteint et reste au dessus d'un certain seuil $c$ s'exprime par $F G([P]>c)$. 
Les propriétés d'oscillation d'une concentration $[P]$ un nombre $n$ de fois, portent sur le nombre d'alternances du signe de la dérivée. Elles s'expriment par la formule

$$
F((d[P] / d t>0) \wedge F((d[P] / d t<0) \wedge F((d[P] / d t>0) \ldots)))
$$

abrégée par oscil $(\mathrm{P}, \mathrm{n})$.

\subsubsection{Algorithme de model-checking avec contraintes}

Sous l'hypothèse que les conditions initiales sont complètement déterminées, les méthodes d'intégration numérique du système d'équations différentielles associées aux règles BIOCHAM, fournissent une trace de simulation discrète (à pas variable) qui constitue une structure de Kripke linéaire, dans laquelle on peut interpréter les formules LTL. De façon à poser des contraintes sur non seulement les valeurs des concentrations mais aussi sur leurs dérivées, on considère des traces de simulation de la forme

$$
\left(<t_{0}, x_{0}, d x_{0} / d t>,<t_{1}, x_{1}, d x_{1} / d t>, \ldots\right)
$$

qui contiennent pour chaque point de temps $t_{i}$, la valeur des concentrations $x_{i}$ et de leurs dérivées $d x_{i} / d t$.

Sous l'hypothèse que les propriétés temporelles peuvent être vérifiées sur un horizon fini, l'algorithme de model-checking avec contraintes pour vérifier une formule $\phi$ consiste alors à effectuer les opérations suivantes :

1) calculer une trace de simulation finie,

2) étiqueter les points de la trace par les sous-formules atomiques de $\phi$ qui sont vraies en ce point ;

3) ajouter les sous-formules de la forme $F \phi_{1}$ aux points prédécesseurs des points étiquetés par $\phi_{1}$,

4) ajouter les sous-formules de la forme $\phi_{1} U \phi_{2}$ aux points prédécesseurs des points étiquetés par $\phi_{2}$ tant qu'ils satisfont $\phi_{1}$

On a $G \phi_{1}=\neg F\left(\neg \phi_{1}\right)$, ce qui se ramène ici à dire qu'on peut étiqueter le dernier état par $G \phi_{1}$ s'il est étiqueté par $\phi_{1}$, puis étiqueter les prédécesseurs d'un état étiqueté $\operatorname{par} G \phi_{1}$, s'il est lui même étiqueté par $\phi_{1}$.

Pour des raisons d'intégration au système BIOCHAM, cet algorithme est implanté en Prolog, de même que les méthodes d'intégration numérique (méthode de RungeKutta à pas variable, et méthode implicite de Rosenbrock pour les systèmes raides).

\subsection{PCTL avec contraintes arithmétiques pour la sémantique des populations}

La logique PCTL (Hansson et al., 1994) remplace essentiellement les opérateurs de choix des chemins $E$ et $A$ de la logique CTL, par un opérateur $P_{\bowtie p}$, désignant la probabilité de réalisation de la formule sous sa portée. Par exemple, $A\left(\psi U \psi^{\prime}\right)$ devient $P_{\geq 1}\left(\psi U \psi^{\prime}\right)$ (imposant que la probabilité que $\psi U \psi^{\prime}$ se réalise soit égale à 1 ). Comme 
dans la section précédente, on s'intéresse à des formules atomiques du premier ordre avec contraintes arithmétiques portant, cette fois-ci, sur des nombres entiers de molécules.

Les algorithmes de model-checking probabiliste existants, comme celui du système PRISM (Kwiatkowska et al., 2004), ne permettent cependant pas de traiter les exemples fortement non déterministes ou pour lesquels les variables ont un grand domaine, ce qui est le cas pour la sémantique de population de BIOCHAM. De meilleurs résultats ont été obtenus avec un algorithme de type Monte-Carlo semblable à celui utilisé dans le système APMC (Hérault et al., 2004). Cet algorithme permet de vérifier une formule LTL avec contraintes, et fournit une probabilité approchée de réalisation de cette formule. Pour ce faire, un ensemble de simulations stochastiques est effectué, en étiquetant les traces de chaque simulation par l'algorithme pour LTL avec contraintes. La probabilité de réalisation est alors estimée par comptage sur l'échantillon. Dans ce cadre, l'opérateur $P_{\bowtie p}$ n'est autorisé qu'en tête de la formule. Ce ne sont donc pas des formules PCTL générales qui sont vérifiées, mais des formules LTL en fournissant une probabilité de réalisation. Le résultat est une probabilité, et non pas la vérification d'une formule comprenant un ensemble de probabilités imbriquées.

\section{Apprentissage de modèles}

Les modèles biologiques sont construits à partir d'expériences réalisées sur des organismes sauvages ou mutés. Ces expériences définissent les propriétés biologiques que les modèles doivent satisfaire. En particulier, les expériences sur les organismes mutés, dans lesquels la synthèse de certaines protéines est bloquée, permettent de discriminer entre différentes hypothèses d'interaction. Dans BIOCHAM, l'utilisation de langages formels pour, à la fois décrire le système (langage de règles), et décrire ses propriétés (spécification en logique temporelle), permet d'appliquer des algorithmes d'apprentissage automatique pour aider à corriger ou compléter un modèle en fonction de nouvelles spécifications.

\subsection{Recherche de règles}

En ce qui concerne la recherche de règles à ajouter ou supprimer au modèle, nous utilisons la sémantique Booléenne de BIOCHAM, et une spécification CTL des propriétés attendues du modèle.

Les formules CTL sont partitionnées en trois classes de façon à guider la recherche pour l'ajout ou la suppression de règles. La classe ECTL est formée des formules CTL qui ne contiennent pas l'opérateur $A$ (c'est-à-dire qui ne contiennent pas d'occurrence positive de $A$ ni d'occurrence négative de $E$ ). La classe ACTL est formée des formules CTL qui ne contiennent pas l'opérateur $E$. On note UCTL l'ensemble des autres formules. 
Proposition 3 Soit $K=(S, R, L)$ et $K^{\prime}=\left(S, R^{\prime}, L\right)$ deux structures de Kripke telles que $R \subseteq R^{\prime}$. Pour toute formule ECTL $\phi$, si $s \not \models_{K^{\prime}} \phi$ alors $s \not \models_{K} \phi$. Pour toute formule ACTL $\phi$, si $s \not \models_{K} \phi$ alors $s \not \models_{K^{\prime}} \phi$.

Preuve 1 Soit $\phi$ une formule ECTL telle que $s \forall_{K^{\prime}} \phi$. Raisonnons par l'absurde et supposons que $s={ }_{K} \phi$, alors il existe un chemin $\pi=s s_{1} s_{2} \ldots$ dans la structure $K$ tel que $\pi=_{K} \phi$ ou $s \models_{K} \phi$. Comme $R \subseteq R^{\prime}$, $\pi$ est aussi un chemin dans la structure $K^{\prime}$, on a donc $\pi \models_{K^{\prime}} \phi$ ou $s \models_{K^{\prime}} \phi$, contradiction.

De même pour une formule ACTL $\phi$, par dualité $\neg \phi$ est une formule ECTL. Si $s \not \forall_{K} \phi$ alors $s \models_{K} \neg \phi$, d'où l'on déduit comme précédemment $s \models_{K^{\prime}} \neg \phi$, c'est-à-dire $s \not K_{K^{\prime}} \phi$.

Cette propositon montre que l'on peut chercher à satisfaire une spécification CTL à partir d'un modèle ne les satisfaisant que partiellement, en supprimant des règles du modèle qui rendent les formules ACTL fausses, et en ajoutant au modèle des règles qui permettent de satisfaire les formules ECTL fausses.

La commande

learn_one_rule(reaction_pattern, spec_CTL)

énumère chaque instance du schéma de règles fourni en premier argument qui, ajoutée au modèle, permet de satisfaire la formule CTL fournie en second argument (ou implicitement avec la commande add_spec). La commande

delete_one_rule(reaction_pattern, spec_CTL)

énumère chaque instance de règle qu'il suffit de retirer au modèle pour satisfaire la formule CTL.

Exemple 3 Dans cet exemple, on retire la règle suivante du modèle de Tyson : delete_rule (Cyclin+Cdc2 $\{\mathrm{p} 1\}=>\operatorname{Cdc} 2 \sim\{\mathrm{p} 1\}-\mathrm{Cyclin} \sim\{\mathrm{p} 1\})$.

Le complexe inactif $\mathrm{Cdc} 2 \sim\{\mathrm{p} 1\}-\mathrm{Cycl}$ in $\sim\{\mathrm{p} 1\}$ ne pouvant plus se former, certaines molécules ne sont plus accessibles. La spécification donnée dans l'exemple 2 n'est donc plus vérifiée.

L'algorithme de recherche de règle propose quatre règles parmi 587 testées. L'ajout de l'une d'elles permet de satisfaire à nouveau la spécification :

biocham: learn_one_rule(elementary_interaction_rules).

(1) $\mathrm{Cyclin}+\mathrm{Cdc} 2 \sim\{\mathrm{p} 1\}=[\mathrm{Cdc} 2]=>\mathrm{Cdc} 2 \sim\{\mathrm{p} 1\}-\mathrm{Cyclin} \sim\{\mathrm{p} 1\}$

(2) Cyclin $+\mathrm{Cdc} 2 \sim\{\mathrm{p} 1\}=[\mathrm{Cyclin}]=>\mathrm{Cdc} 2 \sim\{\mathrm{p} 1\}-\mathrm{Cyclin} \sim\{\mathrm{p} 1\}$

(3) Cyclin $+\mathrm{Cdc} 2 \sim\{\mathrm{p} 1\}=>\operatorname{Cdc} 2 \sim\{\mathrm{p} 1\}-\mathrm{Cyclin} \sim\{\mathrm{p} 1\}$

(4) Cyclin $+\mathrm{Cdc} 2 \sim\{\mathrm{p} 1\}=[\mathrm{Cdc} 2 \sim\{\mathrm{p} 1\}]=>\mathrm{Cdc} 2 \sim\{\mathrm{p} 1\}-\mathrm{Cyclin} \sim\{\mathrm{p} 1\}$ 
La troisième règle correspond à la règle retirée. les autres règles ne sont pas biologiquement plausibles et auraient pu être éliminées d'avance en fournissant plus d'information dans le schéma de règles à chercher.

On définit un algorithme de révision du modèle permettant d'ajouter et retirer plusieurs règles, en fixant une stratégie de recherche des règles à ajouter ou supprimer de façon à satisfaire la spécification. La commande

revise_model(reaction_pattern1, reaction_pattern2, spec_CTL)

énumère de façon heuristique des ensembles d'ajouts et de retraits de règles qui permettent de satisfaire une spécification CTL fournie en argument. L'algorithme utilisé dans BIOCHAM est défini par un système de transitions non-déterministe et backtrackant. L'état d'une recherche est représenté par un triplet $<Q T, Q, R>$ où

- $R$ est l' ensemble courant des règles de réaction.

- $Q T=(\bar{E}, \bar{U}, \bar{A})$ est l'ensemble des formules déjà traitées et satisfaites pour $R$. Ces formules sont triées en 3 groupes : $\bar{E}$ représente les formules ECTL, $\bar{U}$ les formules non classifiables (UCTL) et $\bar{A}$ les formules ACTL.

$-Q=(E, U, A)$ est l'ensemble des formules à traiter triées de la même manière en formules ECTL, UCTL et ACTL.

La spécification $I$ de l'état initial est fixe et on s'intéresse donc à une modification de l'ensemble $R$ des règles du modèle de façon à satisfaire la spécification CTL, ce que l'on note $R \models \phi$ par abus de notation. Les modifications sont cherchées en considérant seulement les instances des schémas de règles fournis pour les ajouts et les retraits. De plus, la recherche des règles à retirer (dans les transitions U' et A' ci-dessous) ne se fait que dans l'ensemble des règles apparaissant dans le contre-exemple fourni par le model-checker.

État initial : $<(\bar{\varnothing}, \bar{\varnothing}, \bar{\varnothing}),(E, U, A), R>$

transition $\mathbf{E}:<(\bar{E}, \bar{U}, \bar{A}),(E \cup\{e\}, U, A), R>\rightarrow<(\overline{E \cup\{e\}}, \bar{U}, \bar{A}),(E, U, A)$, $R>$ si $R=e$

transition $\mathbf{E}^{\prime}:<(\bar{E}, \bar{U}, \bar{A}),(E \cup\{e\}, U, A), R>\rightarrow<(\overline{E \cup\{e\}}, \bar{U}, \bar{A}),(E, U, A)$, $R \cup\{r\}>$ si $R \not \models e$ et $\forall f \in\{e\} \cup \bar{E} \cup \bar{U} \cup \bar{A} K \cup\{r\} \models f$

transition $\mathbf{U}:<(\bar{E}, \bar{U}, \bar{A}),(\varnothing, U \cup\{u\}, A), R>\rightarrow<(\bar{E}, \overline{U \cup\{u\}}, \bar{A}),(\varnothing, U, A)$, $R>$ si $R \models u$

transition $\mathbf{U}^{\prime}:<(\bar{E}, \bar{U}, \bar{A}),(\varnothing, U \cup\{u\}, A), R>\rightarrow<(\bar{E}, \overline{U \cup\{u\}}, \bar{A})$, $(\varnothing, U, A), R \cup\{r\}>$ si $R \not \models u$ et $\forall f \in\{u\} \cup \bar{E} \cup \bar{U} \cup \bar{A} R \cup\{r\} \models f$

transition U" : $<(\bar{E}, \bar{U}, \bar{A}),(\varnothing, U \cup\{u\}, A), R \cup \operatorname{Re}>\rightarrow<(\bar{E}, \overline{U \cup\{u\}}, \bar{A})$, $(\varnothing, U, A), R>\operatorname{si} K: s_{i} \not \models u$ et $\forall f \in\{u\} \cup \bar{E} \cup \bar{U} \cup \bar{A} R \models f$

transition A : $<(\bar{E}, \bar{U}, \bar{A}),(\varnothing, \varnothing, A \cup\{a\}), R>\rightarrow<(\bar{E}, \bar{U}, \overline{A \cup\{a\}})$, $\left(E_{p}, U_{p}, A\right), R>\operatorname{si} R \models a$ 
transition A' : $<\left(\overline{E \cup E_{p}}, \overline{U \cup U_{p}}, \bar{A}\right),(\varnothing, \varnothing, A \cup\{a\}), R \cup R e>\rightarrow<$ $(\bar{E}, \bar{U}, \overline{A \cup\{a\}}),\left(E_{p}, U_{p}, A\right), R>\operatorname{si} R \not \models a, \forall f \in\{u\} \cup \bar{E} \cup \bar{U} \cup \bar{A} R \models f$ et $E_{p} \cup U_{p}$ est l'ensemble des formules rendues non-satisfaites par le retrait des règles $R e$.

Proposition 4 L'algorithme de révision termine et si sa configuration terminale est de la forme $<(\bar{E}, \bar{U}, \bar{A}),(\varnothing, \varnothing, \varnothing), R>$ alors le modèle $R$ vérifie la spécification initiale.

Preuve 2 La terminaison se montre en considérant l'ordre lexicographique sur le couple $\langle a, n>$ où a est le nombre de propriétés ACTL non vérifiées, et n est le nombre de propriétés ECTL ou UCTL non vérifiées. Chaque transition diminue strictement a ou bien laisse a égal et diminue strictement n. La correction de l'algorithme découle du fait que chaque transition préserve l'ensemble des propriétés en les faisant passer entre les ensembles vérifiés et non-vérifiés et ne conserve dans l'ensemble vérifié que des propriétés vraies.

Cet algorithme est toutefois incomplet pour deux raisons. D'une part, la satisfaction d'une formule ECTL ou UCTL n'est recherchée qu'en ajoutant une seule règle au modèle (transitions E' et U'). D'autre part, la complétion de la relation d'accessibilité en une relation totale dans la structure de Kripke associée au modèle, ne garantit pas que les conditions de la proposition 3 sont vérifiées après l'ajout ou le retrait d'une règle. Il s'agit donc d'un algorithme de révision heuristique qui peut échouer dans des cas où la spécification CTL est satisfaisable.

Exemple 4 L'algorithme de révision peut être utilisé pour raffiner un modèle, de façon par exemple à prendre en compte une nouvelle molécule donnée avec une spécification de ses propriétés biologiques. Dans l'exemple du cycle cellulaire, on peut ainsi s'intéresser à la recherche d'une modification du modèle, qui préserve les spécifications précédentes, et fasse intervenir une molécule $\mathrm{X}$ telle que (1) $\mathrm{X}$ ne peut être activée sans la présence de Cdc2-Cyclin $\sim \mathrm{p} 1\}$, et (2) sans $\mathrm{X}, \mathrm{Cyclin} \sim\{\mathrm{p} 1\}$ n'est jamais inactivée ou dégradée :

(1) $\mathrm{AG}(($ ! $(\mathrm{X})) \rightarrow$ checkpoint $(\mathrm{Cdc} 2-\mathrm{Cyclin} \sim\{\mathrm{p} 1\}, \mathrm{X}))$,

(2) $\mathrm{AG}((\mathrm{Cyclin} \sim\{\mathrm{p} 1\}) \rightarrow \operatorname{checkpoint}(\mathrm{X}$, ! (Cyclin $\{\mathrm{p} 1\})))$.

A cette spécification, sont également rajoutées des formules d'accessibilité et d'oscillation relatives à $\mathrm{X}$ :

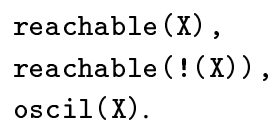

Par défaut, on associe à la molécule $\mathrm{X}$ une dynamique arbitraire avec deux règles simples de synthèse et de dégradation : _=> $\mathrm{X}$ et $\mathrm{X}=>_{-}$.

Ces spécifications ne sont pas vérifiées par le modèle. La commande revise_model, limitée à des règles faisant intervenir $\mathrm{X}$ pour l'ajout de règles, permet de trouver des solutions pour le corriger. La première solution trouvée : 


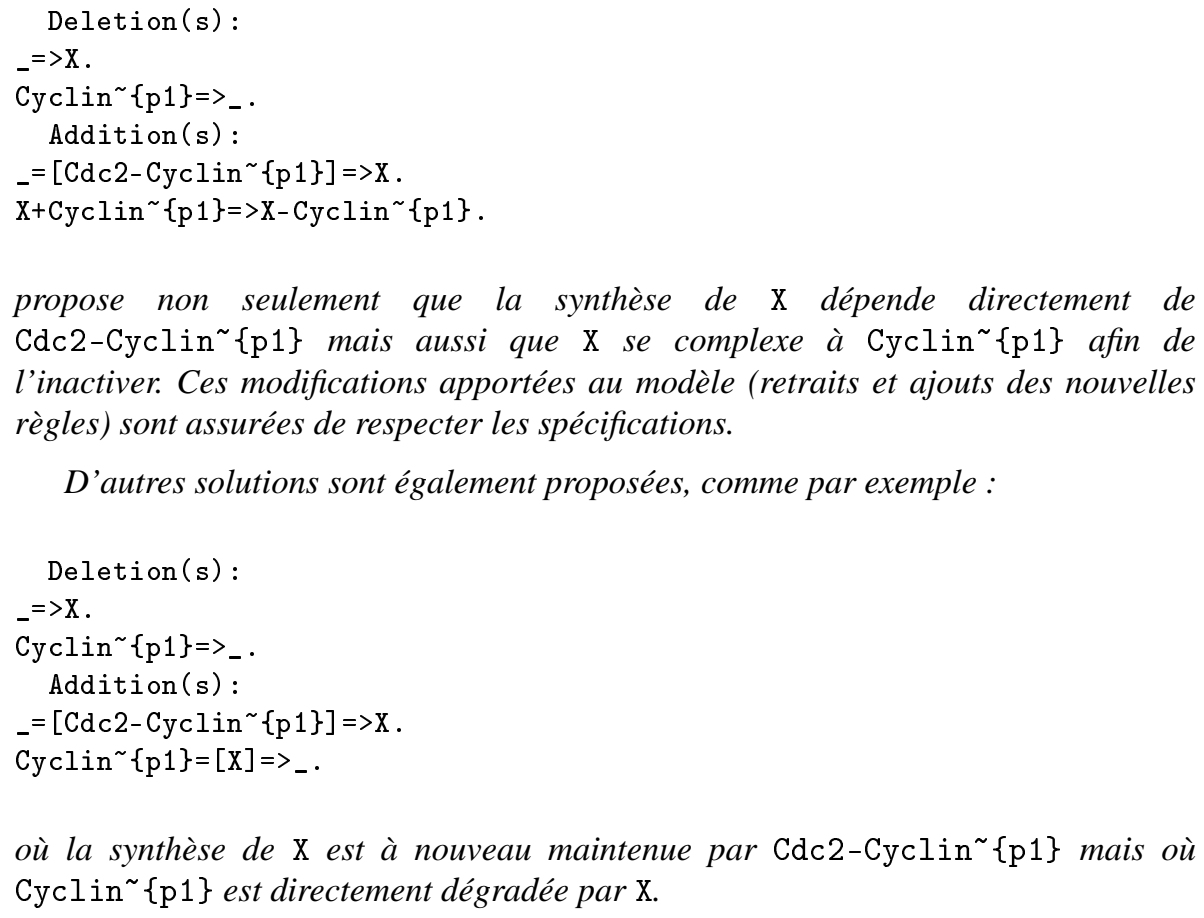

D’autres solutions sont également proposées, comme par exemple :

$\operatorname{Deletion}(\mathrm{s})$ :

- $=>$ X.

Cyclin $\{\mathrm{p} 1\}=>_{-}$.

Addition(s):

$-=[$ Cdc2-Cyclin $\sim$ p1\}] $=>X$.

Cyclin $\{\mathrm{p} 1\}=[\mathrm{X}]=>_{-}$.

où la synthèse de $\mathrm{X}$ est à nouveau maintenue par $\mathrm{Cdc} 2-\mathrm{Cyclin} \sim\{\mathrm{p} 1\}$ mais où Cyclin $\sim \mathrm{p} 1\}$ est directement dégradée par $\mathrm{X}$.

\subsection{Recherche de paramètres}

De la même manière que pour l'apprentissage de règles d'interaction à partir de propriétés CTL, il est possible d'utiliser une spécification LTL avec contraintes arithmétiques pour l'apprentissage de valeurs de paramètres cinétiques. Nous avons développé une méthode énumérative dans laquelle l'espace de recherche, fourni par des bornes sur les valeurs des paramètres, est exploré avec une précision spécifiée par le modélisateur. Pour chaque jeu de valeurs testées, le modèle est simulé et confronté à la spécification LTL.

La recherche de paramètres peut être illustrée à partir du modèle de l'exemple 1 . Deux paramètres sont choisis, $k_{3}$ et $k_{4}$, auxquels sont assignées les valeurs arbitraires 0.01 et 0.01 . La simulation montre que le système atteint un état stationnaire (figure 3 ) or il est censé osciller. Pour y remédier, BIOCHAM recherche, pour chaque paramètre, parmi 20 valeurs différentes et dans un intervalle [0,200], des valeurs telles que Cdc2-Cyclin $\sim$ p1\} oscille au moins 3 fois en 150 unités de temps :

trace_get $([\mathrm{k} 3, \mathrm{k} 4],[(0,200),(0,200)], 20$, oscil (Cdc2-Cyclin $\{\mathrm{p} 1\}, 3), 150)$.

BIOCHAM propose une première solution $\left(k_{3}=10, k_{4}=70\right)$ qui n'est pas satisfaisante car Cdc2-Cyclin $\sim$ p1\} oscille mais avec une très faible amplitude à peine décelable et ne s'active donc pas complètement (figure 4). L'ajout de la contrainte 


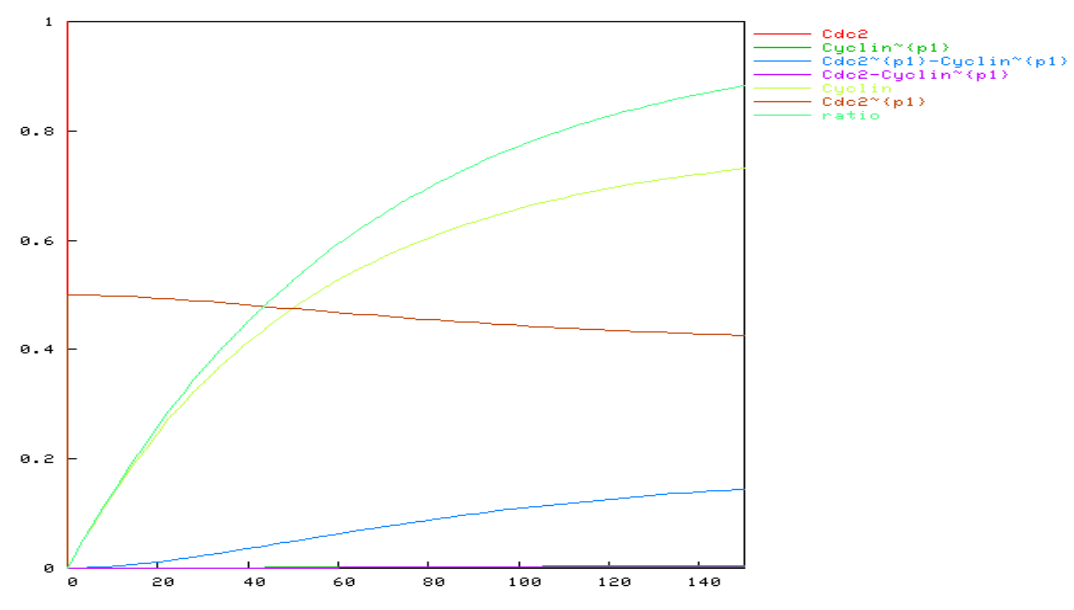

Figure 3. Simulation numérique avec $k_{3}=0.01$ et $k_{4}=0.01$

$\mathrm{F}([\mathrm{Cdc} 2-\mathrm{Cyclin} \sim \mathrm{p} 1\}]>0.15)$ assure que le complexe s'active totalement, à un moment donné, au-dessus de la valeur seuil 0.15, et permet ainsi de raffiner la spécification. L'algorithme de recherche de paramètres trouve alors les valeurs $k_{3}=10$ et $k_{4}=120$. La simulation obtenue (figure 5) est très similaire à celle correspondant aux paramètres publiés $k_{3}=200$ et $k_{4}=180$ (figure 2), ce qui témoigne de la robustesse du modèle initial.

Notons que le nombre de paramètres pour lesquels une valeur est cherchée ainsi que la précision de la recherche influencent le temps de calcul.

D'une certaine manière, le processus d'apprentissage automatise une partie de ce que le modélisateur fait à la main, c'est-à-dire essayer différentes valeurs de paramètres qui correspondent à des formes de courbes d'activité de protéines qu'il souhaite reproduire, et ce dans un intervalle de valeurs de paramètres jugé réaliste. L'algorithme $d$ 'apprentissage permet de tester rapidement des espaces de paramètres après formalisation de l'aspect de la courbe en spécification LTL. Cette méthode est en fait complémentaire d'autres méthodes d'estimation de paramètres telles que l'utilisation de diagrammes de bifurcation.

\section{Conclusion et perspectives}

Le projet BIOCHAM compte parmi les premières expériences d'application des méthodes formelles de vérification et d'apprentissage automatique en modélisation de systèmes biologiques. La sémantique Booléenne est utilisée d'une part pour exprimer des propriétés d'accessibilité, de points de passage obligés, de stationnarité et d'oscillation dans les réseaux d'interaction, et d'autre part pour inférer des règles de réaction 
BIOCHAM 19

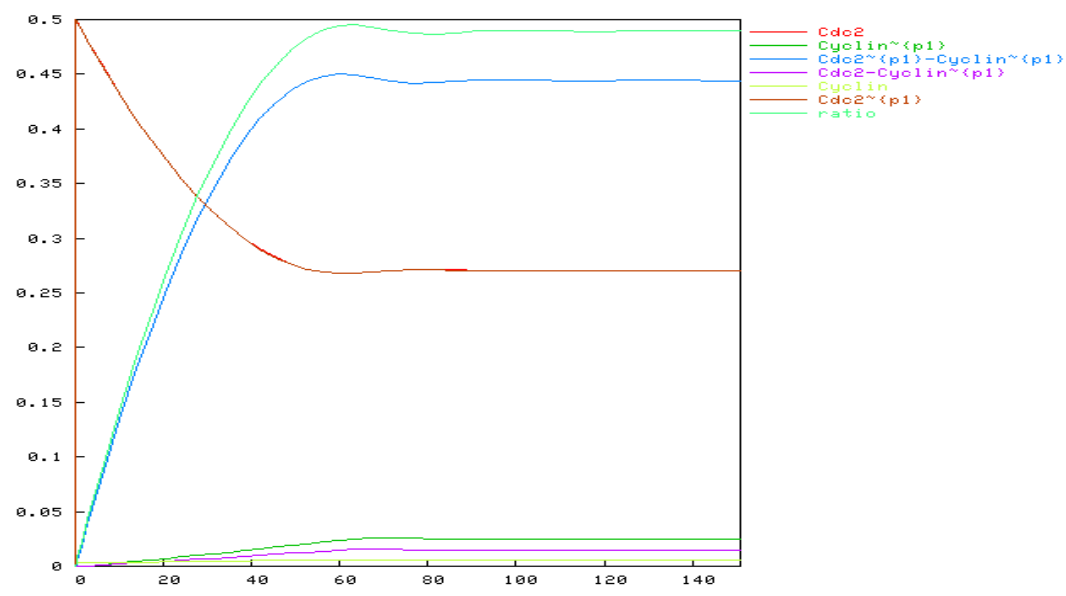

Figure 4. Simulation numérique avec $k_{3}=10$ et $k_{4}=70$

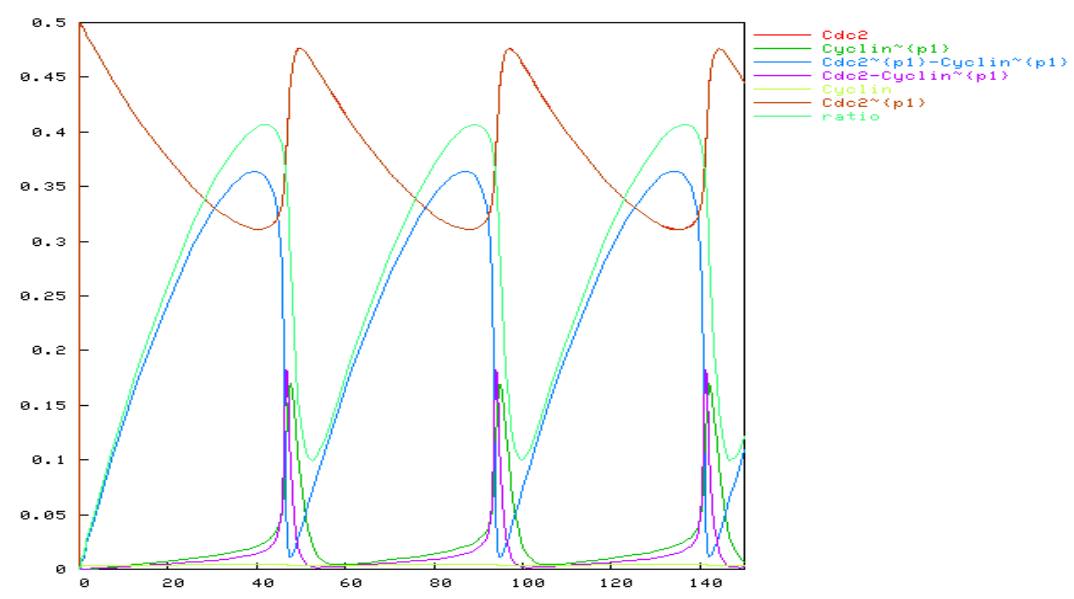

Figure 5. Simulation numérique avec $k_{3}=10$ et $k_{4}=120$

à partir d'une telle spécification. La sémantique des concentrations permet de développer des modèles à dynamique continue. Elle permet aussi d'apprendre des valeurs de paramètres cinétiques à partir des propriétés des courbes numériques correspondant aux observations biologiques formalisées en logique temporelle.

Le cadre formel développé dans BIOCHAM fournit aussi une base pour étudier les questions d'abstraction et de compositionnalité de modèles en biologie des systèmes. 
En particulier nous envisageons d'explorer les techniques d'interprétation abstraite pour formaliser les problèmes de simplification et de raffinement de modèles. L'étude de la compositionnalité vise à faciliter la ré-utilisation de modèles dans différents contextes et à définir des notions d'interfaces dans les modèles ouverts. Ces questions sont actuellement abordées dans l'étude de modèles couplés du cycle cellulaire et du cycle circadien avec l'objectif de contribuer à formuler de nouvelles hypothèses biologiques par ces méthodes.

\section{Bibliographie}

Angelopoulos N., Muggleton S. H., « Machine Learning Metabolic Pathway descriptions using a Probabilistic Relational Representation », Electronic Transactions in Artificial Intelligence, 2002a. also in Proceedings of Machine Intelligence 19.

Angelopoulos N., Muggleton S. H., Slps for Probabilistic Pathways : Modeling and Parameter estimation, Technical Report $n^{\circ}$ TR 2002/12, Department of Computing, Imperial College, London, UK, 2002b.

Antoniotti M., Policriti A., Ugel N., Mishra B., « Model Building and Model Checking for Biochemical Processes », Cell Biochemistry and Biophysics, vol. 38, p. 271-286, 2003.

Batt G., Bergamini D., de Jong H., Garavel H., Mateescu R., « Model Checking Genetic Regulatory Networks using GNA and CADP », Proceedings of the 11th International SPIN Workshop on Model Checking of Software SPIN'2004, Barcelona, Spain, April, 2004.

Bernot G., Comet J.-P., Richard A., Guespin J., « A Fruitful Application of Formal Methods to Biological Regulatory Networks : Extending Thomas' Asynchronous Logical Approach with Temporal Logic », Journal of Theoretical Biology, vol. 229, $n^{\circ}$ 3, p. 339-347, 2004.

Bryant C. H., Muggleton S. H., Oliver S. G., Kell D. B., Reiser P. G. K., King R. D., « Combining Inductive Logic Programming, Active Learning and Robotics to Discover the Function of Genes », Electronic Transactions in Artificial Intelligence, 2001.

Calder M., Vyshemirsky V., Gilbert D., Orton R., « Analysis of Signalling Pathways using the Prism Model Checker », in , G. Plotkin (ed.), CMSB'05 : Proceedings of the third Workshop on Computational Methods in Systems Biology, 2005.

Calzone L., Chabrier-Rivier N., Fages F., Gentils L., Soliman S., « Machine learning biomolecular interactions from temporal logic properties », in, G. Plotkin (ed.), CMSB'05 : Proceedings of the third Workshop on Computational Methods in Systems Biology, 2005.

Cardelli L., « Brane Calculi - Interactions of Biological Membranes », in, V. Danos, , V. Schächter (eds), CMSB'04 : Proceedings of the second Workshop on Computational Methods in Systems Biology, vol. 3082 of Lecture Notes in BioInformatics, Springer-Verlag, p. 257-280, 2004.

Chabrier N., Fages F., « Symbolic model cheking of biochemical networks », in , C. Priami (ed.), CMSB'03 : Proceedings of the first Workshop on Computational Methods in Systems Biology, vol. 2602 of Lecture Notes in Computer Science, Springer-Verlag, Rovereto, Italy, p. 149-162, March, 2003.

Chabrier-Rivier N., Chiaverini M., Danos V., Fages F., Schächter V., « Modeling and querying biochemical interaction networks », Theoretical Computer Science, vol. 325, n 1, p. 25-44, September, 2004a. 
Chabrier-Rivier N., Fages F., Soliman S., « The Biochemical Abstract Machine BIOCHAM », in , V. Danos, , V. Schächter (eds), CMSB'04 : Proceedings of the second Workshop on Computational Methods in Systems Biology, vol. 3082 of Lecture Notes in BioInformatics, Springer-Verlag, p. 172-191, 2004b.

Cimatti A., Clarke E., Enrico Giunchiglia F. G., Pistore M., Roveri M., Sebastiani R., Tacchella A., « NuSMV 2 : An OpenSource Tool for Symbolic Model Checking », Proceedings of the International Conference on Computer-Aided Verification, CAV'02, Copenhagen, Danmark, July, 2002.

Clarke E. M., Grumberg O., Peled D. A., Model Checking, MIT Press, 1999.

Danos V., Laneve C., « Formal Molecular Biology », Theoretical Computer Science, vol. 325, $\mathrm{n}^{\circ} 1$, p. 69-110, 2004.

de Raedt L., Interactive Theory Revision, an inductive Logic Programming Approach, Knowledge-Based Systems, academic press, 1992.

Eker S., Knapp M., Laderoute K., Lincoln P., Meseguer J., Sönmez M. K., « Pathway Logic : Symbolic Analysis of Biological Signaling », Proceedings of the seventh Pacific Symposium on Biocomputing, p. 400-412, January, 2002.

Fages F., Soliman S., Chabrier-Rivier N., « Modelling and Querying Interaction Networks in the Biochemical Abstract Machine BIOCHAM », Journal of Biological Physics and Chemistry, vol. 4, n 2, p. 64-73, October, 2004.

Giaever G. et al., « Functional profiling of the Saccharomyces cerevisiae genome », nature, vol. 418, p. 387-391, Jul, 2002.

Gibson M. A., Bruck J., « A probabilistic model of a prokaryotic gene and its regulation », in , H. Bolouri, , J. Bower (eds), Computational Methods in Molecular Biology : From Genotype to Phenotype, MIT press, chapter 2, 2000.

Gillespie D. T., «General Method for Numerically Simulating Stochastic Time Evolution of Coupled Chemical-Reactions », Journal of Computational Physics, vol. 22, p. 403-434, 1976.

Hansson H., Jonsson B., « A Logic for Reasoning about Time and Reliability », Formal Aspects of Computing, vol. 6, $\mathrm{n}^{\circ}$ 5, p. 512-535, 1994.

Hérault T., Lassaigne R., Magniette F., Peyronnet S., « Approximate Probabilistic Model Checking », Proceedings of the 5th Verification, Model Checking and Abstract Interpretation (VMCAI 2004), vol. 2937 of Lecture Notes in Computer Science, Springer-Verlag, p. 73-84, 2004.

Kanehisa M., Goto S., « KEGG : Kyoto Encyclopedia of Genes and Genomes », Nucleic Acids Research, vol. 28, $\mathrm{n}^{\circ}$ 1, p. 27-30, 2000.

Keseler I. M., Collado-Vides J., Gama-Castro S., Ingraham J., Paley S., Paulsen I. T., PeraltaGil M., Karp P. D., « EcoCyc : a comprehensive database resource for Escherichia coli », Nucleic Acids Research, vol. 33, p. D334-D337, 2005.

Kohn K. W., « Molecular Interaction Map of the Mammalian Cell Cycle Control and DNA Repair Systems », Molecular Biology of the Cell, vol. 10, n 8, p. 703-2734, August, 1999.

Koza J. R., Mydlowec W., Lanza G., Yu J., Keane M. A., « Reverse Engineering of Metabolic Pathways from Observed Data Using Genetic Programming », Proceedings of the 6th Pacific Symposium on Biocomputing (PSB 2001), Hawaii, USA, p. 434-445, January, 2001. 
Kwiatkowska M. Z., Norman G., Parker D., « PRISM 2.0 : A Tool for Probabilistic Model Checking », st International Conference on Quantitative Evaluation of Systems (QEST 2004), IEEE Computer Society, p. 322-323, 2004.

Langley P., Simon H. A., Bradshaw G. L., Zytkow J. M., Scientific Discovery : Computational Explorations of the Creative Processes, MIT Press, Cambridge, MA, February, 1987.

Muggleton S. H., « Inverse Entailment and Progol », New Generation Computing, vol. 13, p. 245-286, 1995.

Phillips A., Cardelli L., « A Correct Abstract Machine for the Stochastic Pi-calculus », Transactions on Computational Systems Biology, to appear. Special issue of BioConcur 2004.

Regev A., Panina E. M., Silverman W., Cardelli L., Shapiro E., « BioAmbients : An Abstraction for Biological Compartments », Theoretical Computer Science, vol. 325, n 1, p. 141-167, September, 2004.

Regev A., Silverman W., Shapiro E. Y., « Representation and simulation of biochemical processes using the pi-calculus process algebra. », Proceedings of the sixth Pacific Symposium of Biocomputing, p. 459-470, 2001.

Schoeberl B., Eichler-Jonsson C., Gilles E., Muller G., « Computational modeling of the dynamics of the MAP kinase cascade activated by surface and internalized EGF receptors », Nature Biotechnology, vol. 20, $\mathrm{n}^{\circ}$ 4, p. 370-375, 2002.

Todorovski L., Džeroski S., « Theory Revision in Equation Discovery », in , K. P. Jantke, , A. Shinohara (eds), Proceedings of the 4th International Conference on Discovery Science, DS 2001, vol. 2226 of Lecture Notes in Artificial Intelligence, Springer-Verlag, Washington, DC, USA, p. 389-400, 2001.

Tomita M. et al., « The E-CELL Project : Towards Integrative Simulation of Cellular Processes », New Generation Computing, vol. 1, n 18, p. 1-12, 2000.

Tyson J. J., « Modeling the cell division cycle : cdc2 and cyclin interactions », Proceedings of the National Academy of Sciences, vol. 88, n 16, p. 7328-7332, August, 1991.

VBI, COPASI's manual. n.d., http ://www. copasi.org/. 